\title{
Aquaporin-6 Expression in the Cochlear Sensory Epithelium Is Downregulated by Salicylates
}

\author{
Paola Perin, ${ }^{1}$ Simona Tritto, ${ }^{1}$ Laura Botta, ${ }^{1}$ Jacopo Maria Fontana, ${ }^{2}$ Giulia Gastaldi, ${ }^{2}$ \\ Sergio Masetto, ${ }^{1}$ Marisa Tosco, ${ }^{3}$ and Umberto Laforenza ${ }^{2}$ \\ ${ }^{1}$ Department of Physiology, Section of General Physiology, University of Pavia, 27100 Pavia, Italy \\ ${ }^{2}$ Department of Physiology, Section of Human Physiology, University of Pavia, Via Forlanini, 6, 27100 Pavia, Italy \\ ${ }^{3}$ Department of Biomolecular Sciences and Biotechnologies, University of Milan, 20133 Milan, Italy
}

Correspondence should be addressed to Umberto Laforenza, lumberto@unipv.it

Received 1 July 2009; Revised 21 September 2009; Accepted 22 October 2009

Academic Editor: Kenichiro Kitamura

Copyright (c) 2010 Paola Perin et al. This is an open access article distributed under the Creative Commons Attribution License, which permits unrestricted use, distribution, and reproduction in any medium, provided the original work is properly cited.

\begin{abstract}
We characterize the expression pattern of aquaporin-6 in the mouse inner ear by RT-PCR and immunohistochemistry. Our data show that in the inner ear aquaporin- 6 is expressed, in both vestibular and acoustic sensory epithelia, by the supporting cells directly contacting hair cells. In particular, in the Organ of Corti, expression was strongest in Deiters' cells, which provide both a mechanical link between outer hair cells (OHCs) and the Organ of Corti, and an entry point for ion recycle pathways. Since aquaporin-6 is permeable to both water and anions, these results suggest its possible involvement in regulating OHC motility, directly through modulation of water and chloride flow or by changing mechanical compliance in Deiters' cells. In further support of this role, treating mice with salicylates, which impair OHC electromotility, dramatically reduced aquaporin-6 expression in the inner ear epithelia but not in control tissues, suggesting a role for this protein in modulating OHCs' responses.
\end{abstract}

\section{Introduction}

Salicylates are a class of nonsteroidal anti-inflammatory drugs (NSAIDs) with analgesic, antipyretic, and anti-inflammatory effects that are known to induce reversible hearing loss and tinnitus [1]. A rationale for salicylate-induced hearing loss is the block of prestin, the molecular motor of outer hair cells $(\mathrm{OHC})$, through competition with intracellular $\mathrm{Cl}^{-}[2-4]$. On the other hand, the mechanism underlying salicylate-induced tinnitus is still unclear, since in the cochlea these drugs, besides prestin [2], also target other systems, among which several players in the stress/inflammation pathways [1]. The stress response affects a large number of targets, among which AQPs [5], the proteins forming water channels in biological membranes [6-8]. Several AQPs have been found in the inner ear, each with its distinct distribution [9-12], but so far only AQP4 is known to affect hearing [13]. The distribution and role of AQP6, however, are less clearly characterized, and may be interesting, given the low permeability to water and high permeability to anions (both increased by low $\mathrm{pH}$ ) of this isoform [14]. Therefore, the function of AQP6 could be that of regulating the flow of anions rather than water, possibly affecting the behavior of outer hair cells (OHCs), which are very sensitive to $\mathrm{Cl}^{-}$modulation [15]. Previous findings show that AQP6 is expressed in human and rat inner ear, but the protein localization is still controversial $[9,11,12]$, possibly due to species differences, which have been observed for other aquaporins $[16,17]$. Whereas in vestibular epithelia AQP6 was found in the apical part of supporting cells both in human [9] and rat [11] in the cochlea AQP6 has been detected in the human Organ of Corti [9] but not in the rat Organ of Corti [11]. In agreement with AQP6 localization in other tissues, AQP6 immunoreactivity in the rat ear appeared limited to intracellular structures, with a negligible labeling in the plasma membranes [11].

In the present paper, we studied AQP6 mRNA expression in the mouse inner ear and its regulation by aspirin, by performing semiquantitative reverse transcription/polymerase chain reaction (RT-PCR), real time RT-PCR (qRT-PCR), immunoblotting and immunohistochemistry. 
TABLE 1: Primer sequences used for semiquantitative and real time reverse transcription/polymerase chain reaction (RT-PCR).

\begin{tabular}{|c|c|c|c|c|c|}
\hline Gene & & Primer sequences & Size (bp) & References & Accession number \\
\hline \multirow{2}{*}{$\beta$-actin* } & Forward & 5'-CAGATCATGTTTGAGACCTT-3' & 509 & {$[18]$} & NM_007393 \\
\hline & Reverse & 5'-CGGATGTCMACGTCACACTT-3' & & & \\
\hline \multirow{2}{*}{ AQP1 } & Forward & 5'-TGCGTTCTGGCCACCACTGAC-3' & 327 & {$[19]$} & NM_007472 \\
\hline & Reverse & 5'-GATGTCGTCAGCATCCAGGTC-3' & & & \\
\hline \multirow{2}{*}{ AQP2 } & Forward & 5'-GTTGCCATGTCTCCTTCCTT-3' & 441 & This study & NM_009699 \\
\hline & Reverse & 5'-GGAACAGCAGGTAGTTGTAG-3' & & & \\
\hline \multirow{2}{*}{ AQP4 } & Forward & 5'-TTTCAAAGGAGTCTGGACTC-3' & 383 & {$[20]$} & NM_009700 \\
\hline & Reverse & 5'-TTCCATGAACCGTGGTGACT-3' & & & \\
\hline \multirow{2}{*}{ AQP6 } & Forward & 5'-CTGCCATGATTGGAACCTCT-3' & 309 & This study & NM_175087 \\
\hline & Reverse & 5'-CTGTGTCCTCAGAGTTTGTC-3' & & & DQ_826418 \\
\hline \multirow{2}{*}{ AQP6a-b } & Forward & 5'-ACTGGCTGTTCCATGAACCC-3' & 727 (a), & {$[14]$} & NM_175087 \\
\hline & Reverse & 5' -AGGAAGTGGCCAGGAGGTACT-3' & 364 (b) & & DQ_826418 \\
\hline \multirow[t]{2}{*}{ AQP7 } & Forward & 5'-TTTGCAGGCGGAGACCTGTT-3' & 437 & {$[21]$} & NM_007473 \\
\hline & Reverse & 5'-CTCTGAGGATCCTGTGGTAT-3' & & & \\
\hline
\end{tabular}

*In real time RT-PCR experiments $\beta$-actin primers were Mm_Actb_2_SG, QuantiTect Primer Assay QT01136772, Qiagen. AQP6a-b, aquaporin-6 variants a and b. (a) and (b): AQP6 variant a and variant $b$.

\section{Methods}

2.1. Animals. C57BL6 mice (20-25 days old) were housed at the animal facility of the Department of Physiology, section of General Physiology, University of Pavia, cared for and sacrificed according to the current European legal Animal Practice requirements.

2.2. Salicylate Treatment. After treatment with saline (i.p., for 3 days) (controls) or aspirin (200 mg/kg/day for 3 days, i.p.) at least 4 animals for each condition in each experiment, the mice were sacrificed 3 hours (treatment) or 24 hours (recovery) after the last injection and processed for RNA isolation or immunolocalization.

2.3. RNA Isolation and Semiquantitative RT-PCR. Total RNA was extracted from the inner ear of at least 4 mice for each condition (saline or aspirin) using the QIAzol Lysis Reagent (QIAGEN, Italy). Single cDNA was synthesized from RNA $(1 \mu \mathrm{g})$ using random hexamers and M-MLV Reverse Transcriptase (Invitrogen S.R.L., Italy). Specific primers for mouse $\beta$-actin and for AQPs were synthesized based on their published sequences (Table 1).

cDNA amplification was performed by GoTaq Flexi DNA Polymerase (Promega, Italy), as previously described [20]. The primers used for amplification are listed in the Table 1. The PCR protocol consisted of an initial denaturation of $5 \mathrm{~min}$ at $96^{\circ} \mathrm{C}$ followed by 35 cycles of denaturation at $96^{\circ} \mathrm{C}$ for $30 \mathrm{~s}$ annealing at $60^{\circ} \mathrm{C}$ for $30 \mathrm{~s}$, and extension at $72^{\circ} \mathrm{C}$ for $30 \mathrm{~s}$. Reverse transcription was always performed both in the presence (positive) or in the absence (negative control) of reverse transcriptase enzyme. The RT-PCR reactions were normalized using $\beta$-actin as an internal standard. First, sequences of the AQP bands were checked by using the Big dye terminator cycle sequencing kit (Applied Biosystem, PE, USA). PCR products were separated on a $3 \%$ Nusieve $(2: 1)$ gel agarose, stained with ethidium bromide, and acquired with the Image Master VDS (Amersham Biosciences Europe). Densitometric analysis of the bands was performed by the Total Lab V 1.11 computer program (Amersham) and the results were expressed as a percentage of the $\mathrm{AQP} / \beta$-actin densitometric ratio. The molecular weight of the PCR products was compared with the DNA molecular weight marker VIII (Roche Molecular Biochemicals, Italy).

2.4. $q R T-P C R$. qPCR was performed in triplicate using $1 \mu \mathrm{g}$ cDNA, obtained as above indicated, and specific primers (Table 1). MESA GREEN qPCR MasterMix Plus (Eurogentec) was used according to the manufacturer instruction and qPCR performed using Rotor Gene 6000 (Corbett). The conditions were as follows: initial denaturation at $95^{\circ} \mathrm{C}$ for $5 \mathrm{~min}, 40$ cycles of denaturation at $95^{\circ} \mathrm{C}$ for $30 \mathrm{~s}$; annealing at $60^{\circ} \mathrm{C}$ for $30 \mathrm{~s}$, and elongation at $72^{\circ} \mathrm{C}$ for $40 \mathrm{~s}$. The $\mathrm{qRT}$-PCR reactions were normalized using $\beta$-actin as housekeeping gene (Mm_Actb_2_SG, QuantiTect Primer Assay QT01136772, Qiagen). Melting curves were generated to detect the melting temperatures of specific products immediately after the PCR run. Relative mRNA levels were determined by comparative quantitation (Corbett) and the results expressed as fold change. 
2.5. Immunoblotting. Immunoblotting was performed as previously described [22]. Mouse kidneys were homogenized with an ULTRA-TURRAX homogenizer (IKA) in a solution containing; $100 \mathrm{mM} \mathrm{NaCl}, 1 \mathrm{mM}$ EDTA, $10 \mathrm{mM}$ Tris- $\mathrm{HCl}$, $\mathrm{pH} 6.8$, and $0.1 \mathrm{mg} / \mathrm{mL}$ PMSF, and treated as previously described [23]. Lanes were loaded with $30 \mu \mathrm{g}$ proteins, subjected to $12.5 \%$ SDS-polyacrilamide gel electrophoresis and transferred to Hybond ECL nitrocellulose membranes (Amersham) by electroelution. After $3 \mathrm{hrs}$ blocking with Tris buffered saline (TBS) containing 5\% nonfat dry milk and 1\% Triton X-100 (blocking solution), membranes were incubated overnight with affinity purified antibody to AQP6, diluted 1: 800 in blocking solution. Antibodies directed against the C-terminal peptide of rat AQP6 with an additional N-terminal cysteine were prepared according to Yasui et al. [24]. Membranes were washed and incubated for $1 \mathrm{~h}$ with peroxidase-conjugated goat antirabbit immunoglobulin G (1 : 120000 in blocking solution) (Amersham Biosciences Europe, Italy). The bands were detected with an ECL Advance western blotting detection system (Amersham Biosciences Europe, Italy). Control experiments (not shown) were performed, by preadsorbing the antibody with a 20 fold molar excess of the immunizing peptide and by incubating the blots with pre-immune rabbit serum. ChemiBlot Molecular Weight Markers were used to accurately estimate the molecular weight and as a positive control for the immunoblot (Chemicon International, Inc., CA, USA).

2.6. Immunohistochemistry. C57BL6 mice were anesthetized with 2-bromo-2-chloro-1,1,1-trifluoroethane and intracardially perfused with acetate-buffered $4 \%(\mathrm{w} / \mathrm{v})$ formalin. Temporal bones were rapidly removed, postfixed for $1 \mathrm{~h}$, decalcified for at least 1 week in EDTA $4 \mathrm{M}$ and successively processed into paraffin. Serial paraffin sections $(5 \mu \mathrm{m})$ were brought to water and then washed with PBS. Sections were successively treated with $3 \%(\mathrm{w} / \mathrm{v}) \mathrm{H}_{2} \mathrm{O}_{2}$ for $20 \mathrm{~min}$ at room temperature $\left(22^{\circ} \mathrm{C}\right)$ to block endogenous peroxidases. After washing for 5 min with PBS, sections were blocked with 3\% $(\mathrm{w} / \mathrm{v})$ BSA in PBS for $60 \mathrm{~min}$ at room temperature. Sections were incubated overnight at $4^{\circ} \mathrm{C}$ with rabbit anti-AQP6 [22] diluted at $1: 500$ in PBS containing 1\% BSA. After three 5minute washes with PBS containing 1\% BSA, sections were treated according to the Labeled Streptavidin Biotin (LSAB) Method (Universal LSAB kit, Dako Italia S.p.A., Italy) and washed three times with PBS containing 1\% BSA. The reaction was visualized by incubation with DakoCytomation Liquid DAB (DakoCytomation, Milan, Italy). Sections were counterstained with haematoxylin and mounted on DPX (Merck Eurolab, Milan, Italy). Control experiments were performed simultaneously using pre-immune serum or omitting the primary antibody.

Immunostained slides were examined by light microscopy using an Olympus BX41 and the digital images captured with the Nikon DS-Fil digital camera using Nis Element F Imaging Software.

2.7. Statistics. All data are expressed as means \pm SEM. The significance of the differences of the means was evaluated with one-way ANOVA followed by Newman-Keuls's $Q$ test,

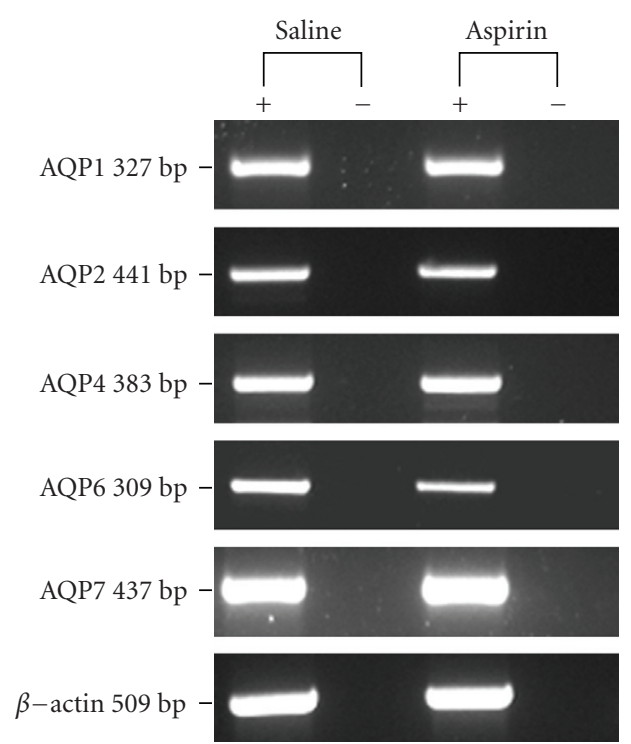

FIGURE 1: Effect of aspirin on aquaporin-1, -2, -4, -6, and -7 (AQP1, $2,4,6,7)$ mRNA expression in the mouse inner ear. Representative semiquantitative RT-PCR with specific primers for AQP1, 2, 4, 6,7 in control (saline) and aspirin treated mice (see Methods). Reverse transcription was performed in the presence $(+)$ or absence $(-)$ of reverse transcriptase enzyme. AQP mRNA expression was normalized to $\beta$-actin. The 327, 441, 383, 309, 437, and $509 \mathrm{bp}$ bands correspond to the AQP-3, $-4,-8$ and $\beta$-actin-specific PCR products, respectively. Similar results were obtained from at least four different mouse RNA extracts.

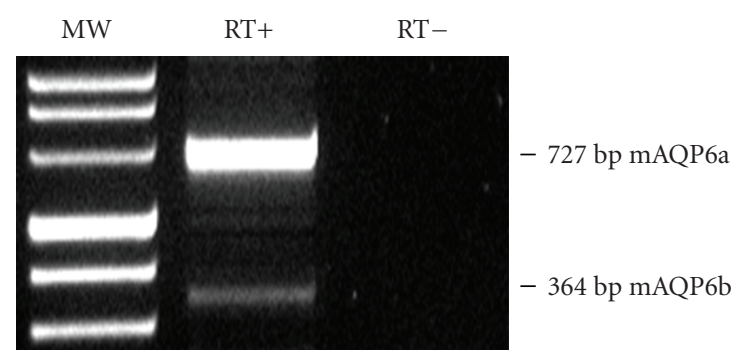

FIGURE 2: RT-PCR analysis of AQP6 splice variants in the mouse inner ear. Representative RT-PCR with specific primers designed to amplify $727 \mathrm{bp}$ and $364 \mathrm{bp}$ fragments for mAQP6a and mAQP6b, respectively, [14]. Reverse transcription was performed in the presence $(\mathrm{RT}+)$ or absence (RT-) of reverse transcriptase enzyme. Similar results were obtained from at least four different mouse RNA extracts.

or Student's $t$ test. All statistical tests were carried out with GraphPad Prism 4.00, 2003.

\section{Results}

3.1. RT-PCR. Expression of AQPs in the mouse inner ear was first verified by semiquantitative RT-PCR after three days of treatment with aspirin or saline (control condition). The expression of each AQP was normalized to that of the housekeeping gene $\beta$-actin. Single bands of the expected size 


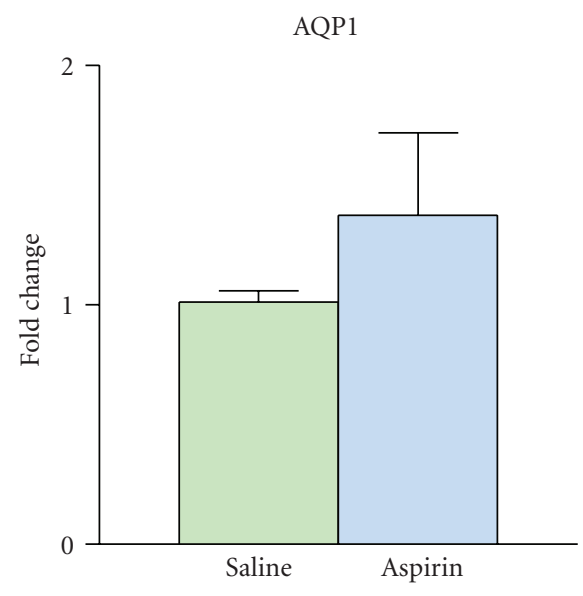

(a)

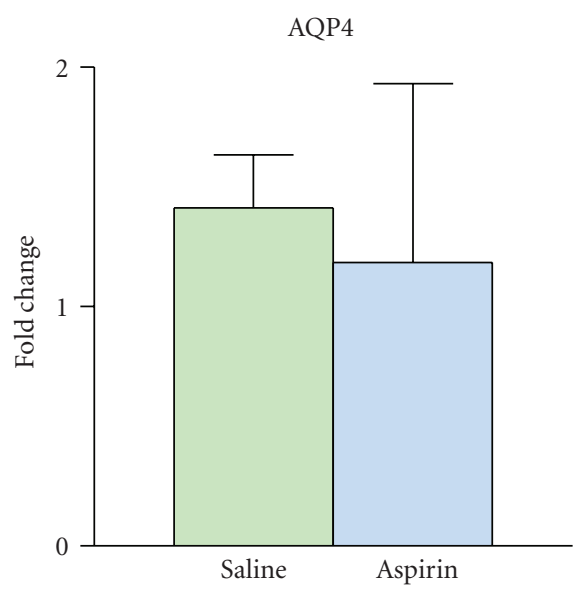

(c)

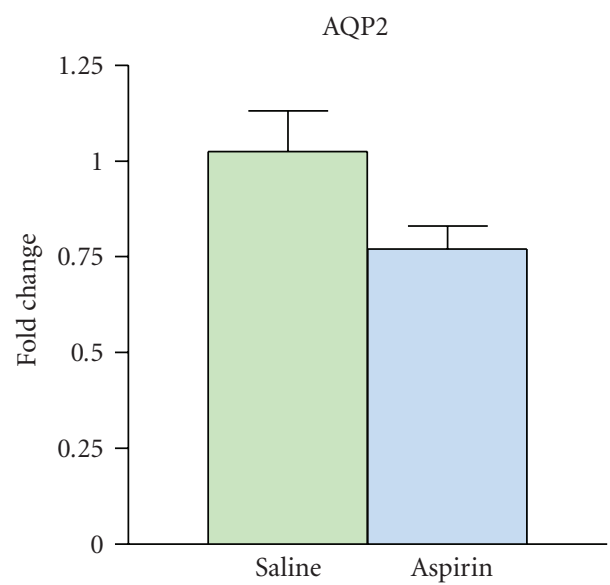

(b)

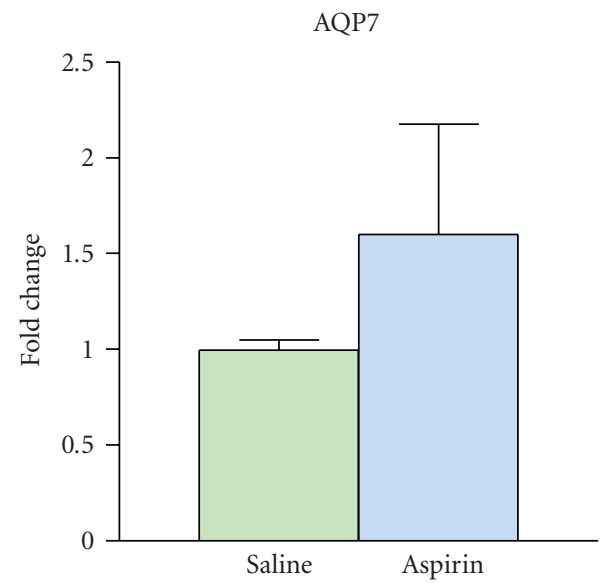

(d)

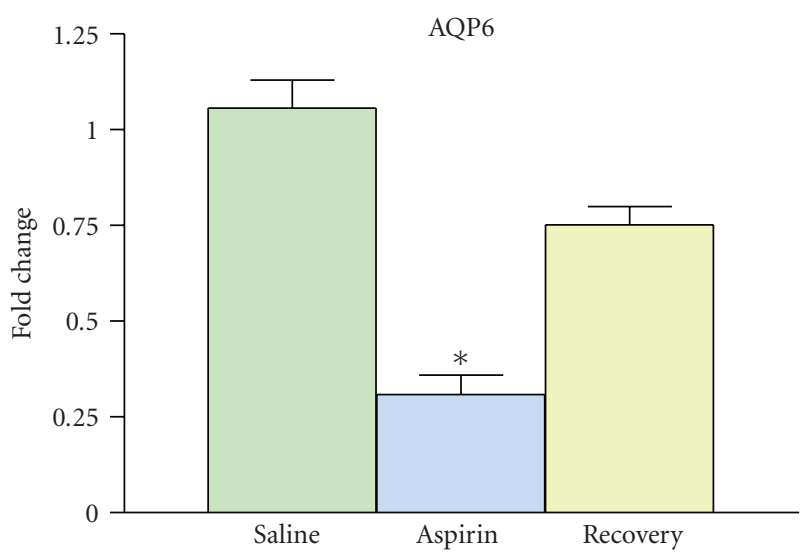

(e)

FIGURE 3: Effect of aspirin treatment on aquaporin (AQP1, 2, 4, 6, 7) mRNA expression in mouse inner ear. Quantitative real-time RT-PCR of total RNA was performed in mouse inner ear mRNA extract under three different conditions: saline (controls), aspirin (mice treated for 3 days and sacrificed 3 hours after the last injection) recovery (treated for 3 days and sacrificed 24 hours after the last injection). Specific primers for AQP1, 2, 4, 6, 7 were used and qRT-PCR performed as indicated (see Methods). The relative mRNA levels were determined by comparative quantitation (Corbett), the values obtained were normalized to the corresponding $\beta$-actin level and the results expressed as fold change. Results indicate that mRNA expression level of AQP6 was significantly lower in aspirin treated mice. Bars represent the mean \pm S.E.M. of 4-8 different experiments each from different RNA extracts. $*, P<.05$ versus saline and recovery (one-way ANOVA followed by Newman-Keuls's Q test). 
of cDNA fragments were amplified $(327,441,383,309,437$ and $509 \mathrm{bp}$ for AQP1, 2, 4, 6, 7 and $\beta$-actin, resp.). The results of agarose gel electrophoresis of representative PCR reaction products are shown in Figure 1. Negative controls were performed by omitting the reverse transcriptase. Only AQP6 mRNA expression was clearly affected by aspirin treatment, while other AQPs were unchanged (Figure 1). Densitometric analysis of the bands showed that AQP6 transcript was significantly reduced (means \pm S.E.M. of AQP6/ $\beta$-actin densitometric ratio): saline, $161 \pm 15 \%$; aspirin, $111 \pm 13 \%$ ( ${ }^{*} P<.043$ Student's $t$ test).

Recently, Yasui and coworkers [14] found two AQP6 splice variants in the mouse kidney: AQP6a (similar to the rat AQP6), and AQP6b, found also in the mouse cerebellum. We performed RT-PCR using specific primers designed to differentiate between the two variants as in [14] and found both variants expressed in the ear (Figure 2).

3.2. $q R T-P C R$. The effect of aspirin treatment on the expression of AQP mRNAs in the mouse inner ear was then investigated by quantitative real time RT-PCR. Results showed that aspirin treatment significantly decreases AQP6 mRNA expression (about 70\%, Figure 3), while no significant changes were observed for AQP1, 2, 4 and 7 transcripts under this condition. Moreover, the results showed a significant recovery of mRNA AQP6 expression 24 hours after the last treatment with aspirin (Figure 3). To investigate the specificity of aspirin effect, we checked AQP6 mRNA levels in the kidney and in the small intestine. In these organs, no significant changes of AQP6 transcript expression were observed: small intestine, $0.883 \pm 0.001$ (saline) and $0.791 \pm$ 0.125 (aspirin); kidney, $1.01 \pm 0.03$ (saline) and $0.96 \pm 0.09$ (aspirin) (fold change; $P>.5$, Student's $t$ test).

3.3. Immunoblotting. Immunoblots of mouse kidney homogenates have been performed to test the antibody used for immunohistochemistry. The results revealed the expected bands (Figure 4), consistent with published data [14].

3.4. Immunohistochemistry. Cellular localization of AQP6 was investigated with immunohistochemistry using affinitypurified antibodies against mouse and rat AQP6. AQP6 was expressed in both cochlear (Figures 5(a), 5(c) and 6(a)) and vestibular (Figure 6(b)) epithelia, and in neuronal somata in the spiral ganglion (Figure 5(b)); faint or no labelling was noted in the stria vascularis (not shown). In the Organ of Corti (Figure 5(a), 5(c)), labelling was most intense and consistent in Deiters' cells, which were labelled throughout their length (Figure 5(a), 5(c)), and also present at inner phalangeal cells and pillars of Corti (Figure 5(a)). Controls in which the primary antibody was replaced by the pre-immune serum show an absence of labelling (Figure 5(d)). No clear labelling was observed in hair cells (Figure 5(a) and 5(c)). In vestibular epithelia (Figure 6(b)), labelling was evident, mainly in supporting cells.

After treatment with aspirin (see Methods), labelling was strongly reduced or lost in both the Organ of Corti (Figure 6(c)) and vestibular epithelia (Figure 6(d)). Reduction in ganglion cells was less evident (not shown).

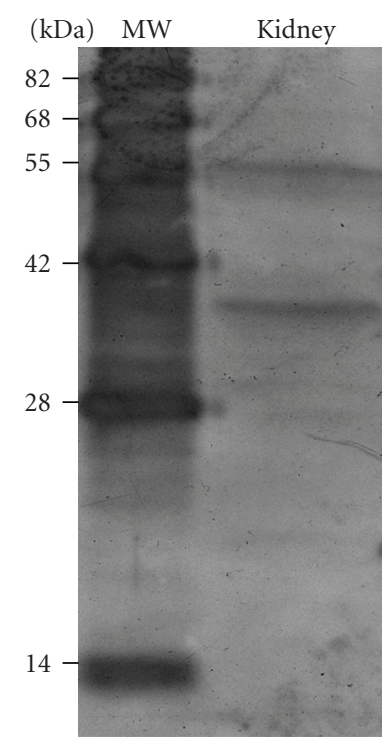

FIGURE 4: AQP6 protein expression in mouse kidney homogenate. Immunoblot of mouse kidney total homogenate was performed as described (see Methods). Two bands of about 55 and $36 \mathrm{kDa}$ were observed. Similar results were obtained from at least four separate experiments. MW, molecular weight markers.

\section{Discussion}

In the present paper, we characterize for the first time the expression pattern of AQP6 in the mouse ear sensory epithelia and its regulation by salicylates.

Our data agree with previous studies in human [9] and rat [11] showing strong AQP6 expression in the supporting cells of vestibular epithelia. In the cochlea, on the other hand, our results disagree with previous localization in the rat, where labelling was seen in the stria vascularis but not in the organ of Corti. Although some discrepancies could be ascribed to differences in species and antibodies used, it is also possible that the different labelling observed in the organ of Corti results from variations in the modulation set point of expression, as our salicylate experiments show.

Our data show that AQP6 is expressed in the organ of Corti, and particularly in Deiters' cells. This pattern of expression suggests a possible role for AQP6 in regulating OHC electromotility, which involves both water and chloride flow, or in modulating Deiters' cell stiffness, which would affect OHC action on basilar membrane movement [15]. In facts, AQP6 displays interesting properties, and it is still debated whether its main function is that of an anion channel, a water channel, or both [25]. Moreover, although originally thought to be only intracellular [26], recent data show that AQP6 can bind calmodulin with its $\mathrm{N}$ terminal domain [27], which is responsible for its subcellular localization [26]. It is therefore possible that stimuli that increase cytoplasmic $\mathrm{Ca}^{2+}$ (such as purinergic signalling in Deiters' cells [28]), induce AQP6 traslocation to the plasma membrane, similar to what vasopressin does to AQP2 [29]. 


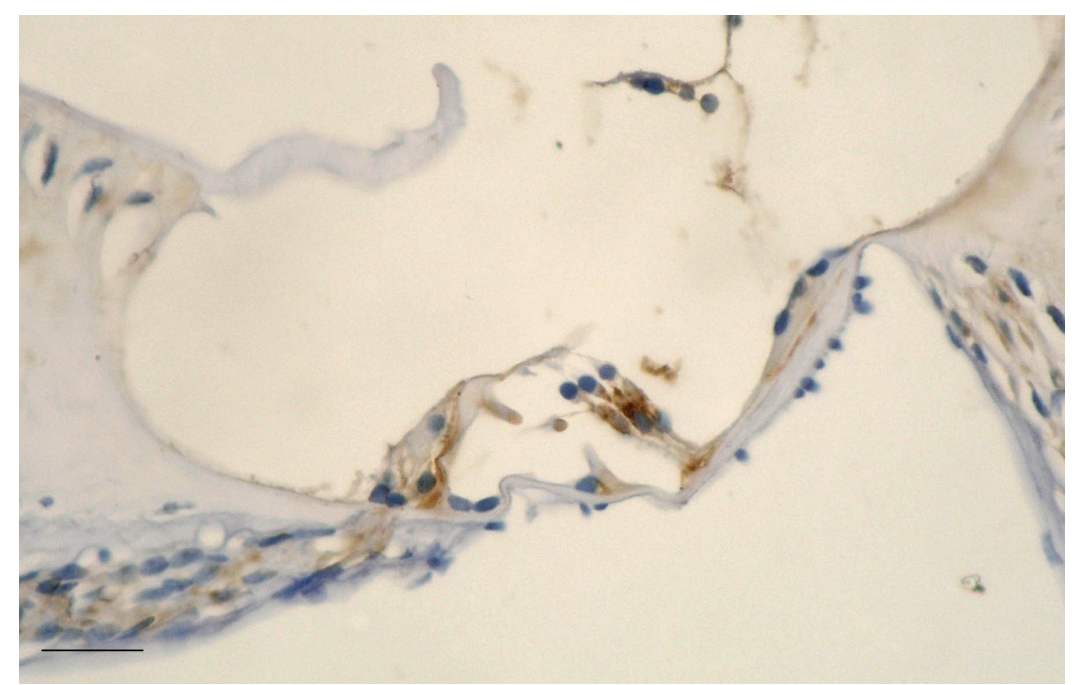

(a)

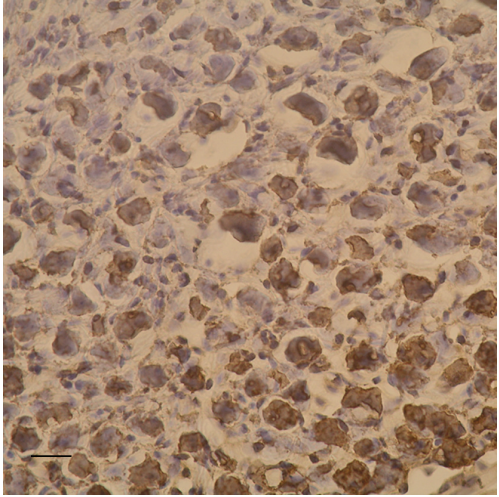

(b)
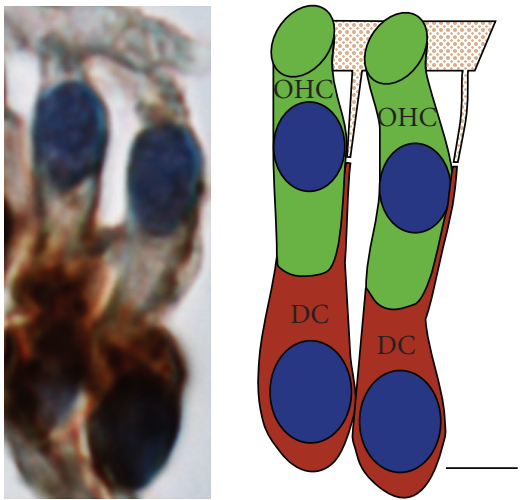

(c)

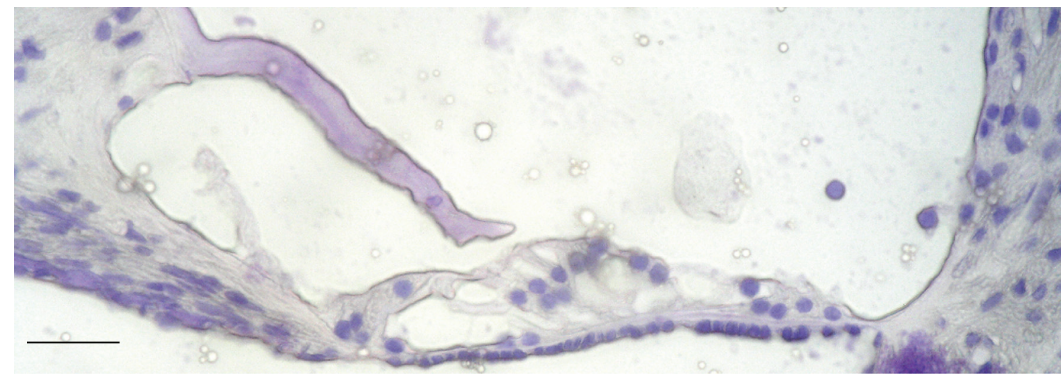

(d)

FIGURE 5: Immunohistochemical localization of the aquaporin-6 protein in the mouse inner ear. Paraffin embedded cochlear sections were labeled with anti-AQP6 antibody. In the cochlea AQP6 staining was observed in the OHC region (a), especially in Deiters' cells (a), (c). Lower signal was observed in inner phalangeal cells (a) and spiral ganglion (b). (c): higher magnification detail of the organ of Corti in the OHC region. The scheme on the right depicts the complex arrangement of Deiters' cells (DC, brown) and outer hair cells (OHC, green). Nuclei are shown in blue. The lighter apical part of Deiters' cells is the terminal lamina, which was not labeled. Deiters' cell stalk and body were instead clearly labeled. Given the complex shape of these cells, it appears difficult to localize AQP6 to cytoplasmic or membrane compartments. Control sections treated with pre-immune serum show an absence of labelling (d). The micrographs are representative of four separate experiments. Scale bar: $20 \mu \mathrm{m}$ (a), (b), (d), $10 \mu \mathrm{m}$ (c).

To further explore this role, we treated mice with salicylates, which are known to impair OHC electromotility, and observed variations in the expression of AQP6 after treatment. AQP6 mRNA expression and protein labeling of the organ of Corti are dramatically reduced after treatment with aspirin. The reduction is quite rapid, being evident already at $3 \mathrm{hrs}$ after the last day of treatment and reversed 24 hours after treatment has ended. 


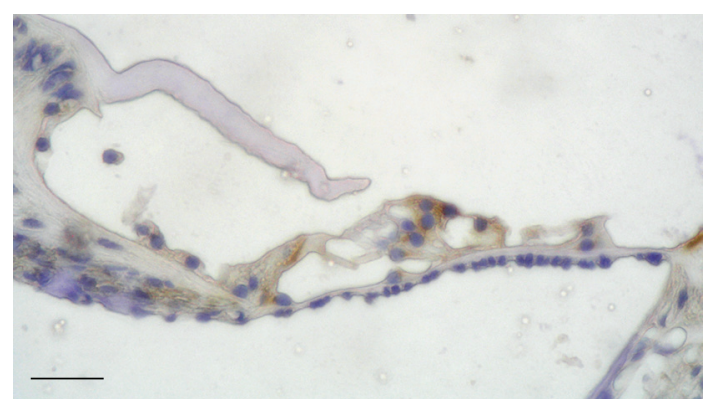

(a)

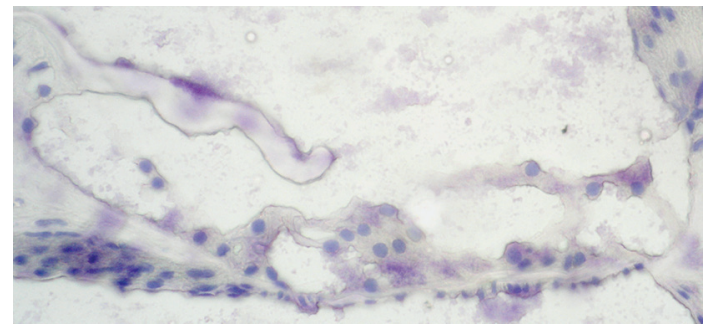

(c)

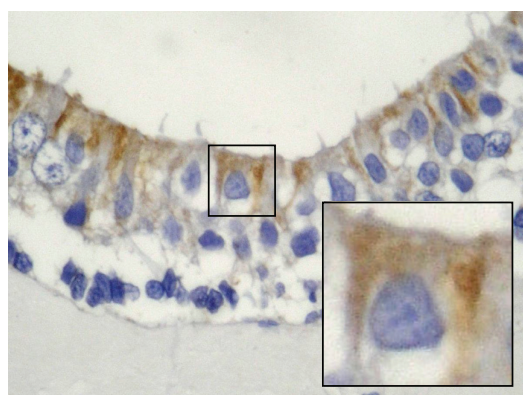

(b)

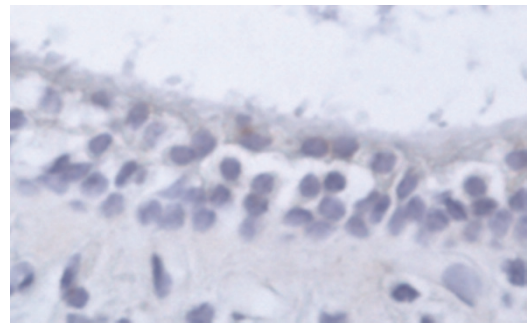

(d)

FIGURE 6: Effect of aspirin treatment on aquaporin-6 protein in the mouse inner ear. Mice were treated with saline (a), (b) and salicylate (c), (d) as described in Methods. Same conditions as Figure 5. In both the organ of Corti (a), (c) and vestibular epithelia (b), (d), AQP6 labeling was lost or strongly attenuated. In vestibular epithelia of saline treated mice, AQP6 was present, possibly in supporting cells. Inset in (b) shows a $2.5 \times$ magnification of the region in the box displaying labeling of the apical part of the hair cell (above the nucleus in the inset) and of the thin supporting cells (whose nuclei are deeper in the epithelium and therefore not in the inset). The micrographs are representative of four separate experiments. Scale bar: $20 \mu \mathrm{m}((\mathrm{a}),(\mathrm{c})), 10 \mu \mathrm{m}((\mathrm{b}),(\mathrm{d}))$.

These results appear interesting because salicylates are well known for their reversible ototoxic effect but the pathways through which they act are still largely unclear. Since prestin and AQP6 appear to be expressed by different cell types, the link between their modulations may be complex.

One possible link between OHC block and AQP6 downregulation in Deiters' cells is COX-2. In fact, this enzyme has been found to be involved in the downregulation of AQP2 in the kidney [30], and is expressed in Deiters' cells, where it is modulated by sound conditioning, possibly through mechanically sensitive pathways [31]. This enzyme could be modulated by salicylates (and by unknown physiological processes) in vestibular epithelia as well (where neither prestin nor motility are present), thus explaining AQP6 downregulation in these organs. Moreover, it is interesting to note the absence of downregulation in small intestine and in the kidney epithelia, suggesting the presence of different modulation mechanisms in different organs.

\section{Conclusions}

In the present paper, we characterize the expression pattern of AQP6 in the mouse inner ear sensory epithelia. Our data show that AQP6 is expressed in the organ of Corti, and in particular in Deiters' cells. This suggests a possible role for AQP6 in regulating OHC motility, through modulation of water and chloride flow or of Deiters' cell mechanical compliance. To further explore this role, we treated mice with salicylates, which impair OHC electromotility, and observed variations in the expression of AQP6 after treatment. AQP6 mRNA expression and protein labeling of the organ of Corti are dramatically reduced already at $3 \mathrm{hrs}$ after the last day of treatment with aspirin. Similar reduction is observed in vestibular epithelia, but not in control tissues (small intestine and kidney). These results suggest that AQP6 downregulation is not due to motility/prestin-dependent pathways, but may affect OHCs' ionic environment and/or their mechanical response through changes in coupling to the Organ of Corti.

\section{Acknowledgments}

This research was funded in part by AIT (Associazione Italiana Tinnitus) ONLUS.

\section{References}

[1] Y. Cazals, "Auditory sensori-neural alterations induced by salicylate," Progress in Neurobiology, vol. 62, no. 6, pp. 583-631, 2000.

[2] D. Oliver, D. Z. Z. He, N. Klöcker, et al., "Intracellular anions as the voltage sensor of prestin, the outer hair cell motor protein," Science, vol. 292, no. 5525, pp. 2340-2343, 2001.

[3] S. Kakehata and J. Santos-Sacchi, "Effects of salicylate and lanthanides on outer hair cell motility and associated gating charge," The Journal of Neuroscience, vol. 16, no. 16, pp. 48814889, 1996. 
[4] J. Zheng, W. Shen, D. Z. Z. He, K. B. Long, L. D. Madison, and P. Dallos, "Prestin is the motor protein of cochlear outer hair cells," Nature, vol. 405, no. 6783, pp. 149-155, 2000.

[5] M. Fukushima, T. Kitahara, Y. Fuse, Y. Uno, K. Doi, and T. Kubo, "Changes in aquaporin expression in the inner ear of the rat after i.p. injection of steroids," Acta Oto-Laryngologica, vol. 124, no. 553s, pp. 13-18, 2004.

[6] P. Agre, L. S. King, M. Yasui, et al., "Aquaporin water channels-from atomic structure to clinical medicine," Journal of Physiology, vol. 542, no. 1, pp. 3-16, 2002.

[7] A. S. Verkman, "Mammalian aquaporins: diverse physiological roles and potential clinical significance," Expert Reviews in Molecular Medicine, vol. 10, article e13, 2008.

[8] K. Ishibashi, "New members of mammalian aquaporins: AQP10-AQP12," Handbook of Experimental Pharmacology, no. 190, pp. 251-262, 2009.

[9] I. A. Lopez, G. Ishiyama, M. Lee, R. W. Baloh, and A. Ishiyama, "Immunohistochemical localization of aquaporins in the human inner ear," Cell and Tissue Research, vol. 328, no. 3, pp. 453-460, 2007.

[10] D. Huang, P. Chen, S. Chen, M. Nagura, D. J. Lim, and X. Lin, "Expression patterns of aquaporins in the inner ear: evidence for concerted actions of multiple types of aquaporins to facilitate water transport in the cochlea," Hearing Research, vol. 165, no. 1-2, pp. 85-95, 2002.

[11] D. Taguchi, T. Takeda, A. Kakigi, T. Okada, R. Nishioka, and H. Kitano, "Expression and immunolocalization of aquaporin-6 (Aqp6) in the rat inner ear," Acta Oto-Laryngologica, vol. 128, no. 8, pp. 832-840, 2008.

[12] M. Fukushima, T. Kitahara, Y. Uno, Y. Fuse, K. Doi, and T. Kubo, "Effects of intratympanic injection of steroids on changes in rat inner ear aquaporin expression," Acta OtoLaryngologica, vol. 122, no. 6, pp. 600-606, 2002.

[13] J. Li and A. S. Verkman, "Impaired hearing in mice lacking aquaporin-4 water channels," The Journal of Biological Chemistry, vol. 276, no. 33, pp. 31233-31237, 2001.

[14] H. Nagase, J. Ågren, A. Saito, et al., "Molecular cloning and characterization of mouse aquaporin 6," Biochemical and Biophysical Research Communications, vol. 352, no. 1, pp. 1216, 2007.

[15] G. I. Frolenkov, "Regulation of electromotility in the cochlear outer hair cell," Journal of Physiology, vol. 576, no. 1, pp. 43-48, 2006.

[16] Y. Miyabe, T. Kikuchi, and T. Kobayashi, "Comparative immunohistochemical localizations of aquaporin-1 and aquaporine-4 in the cochleae of three different species of rodents," Tohoku Journal of Experimental Medicine, vol. 196, no. 4, pp. 247-257, 2002.

[17] J. Loffing, D. Loffing-Cueni, A. Macher, et al., "Localization of epithelial sodium channel and aquaporin-2 in rabbit kidney cortex," American Journal of Physiology, vol. 278, no. 4, pp. F530-F539, 2000.

[18] G. Calamita, A. Mazzone, A. Bizzoca, et al., "Expression and immunolocalization of the aquaporin- 8 water channel in rat gastrointestinal tract," European Journal of Cell Biology, vol. 80, no. 11, pp. 711-719, 2001.

[19] K. Oshio, D. K. Binder, B. Yang, S. Schecter, A. S. Verkman, and G. T. Manley, "Expression of aquaporin water channels in mouse spinal cord," Neuroscience, vol. 127, no. 3, pp. 685-693, 2004.

[20] U. Laforenza, E. Cova, G. Gastaldi, et al., "Aquaporin-8 is involved in water transport in isolated superficial colonocytes from rat proximal colon," The Journal of Nutrition, vol. 135, no. 10, pp. 2329-2336, 2005.
[21] U. Laforenza, G. Gastaldi, M. Grazioli, et al., "Expression and immunolocalization of aquaporin-7 in rat gastrointestinal tract," The Biology of the Cell, vol. 97, no. 8, pp. 605-613, 2005.

[22] U. Laforenza, G. Gastaldi, M. Polimeni, et al., "Aquaporin-6 is expressed along the rat gastrointestinal tract and upregulated by feeding in the small intestine," BMC Physiology, vol. 9, article 18, pp. 1-12, 2009.

[23] U. K. Laemmli, "Cleavage of structural proteins during the assembly of the head of bacteriophage T4," Nature, vol. 227, no. 5259, pp. 680-685, 1970.

[24] M. Yasui, T.-H. Kwon, M. A. Knepper, S. Nielsen, and P. Agre, "Aquaporin-6: an intracellular vesicle water channel protein in renal epithelia," Proceedings of the National Academy of Sciences of the United States of America, vol. 96, no. 10, pp. 5808-5813, 1999.

[25] M. Yasui, "pH regulated anion permeability of aquaporin-6," Handbook of Experimental Pharmacology, vol. 190, pp. 299308, 2009.

[26] E. Beitz, K. Liu, M. Ikeda, W. B. Guggino, P. Agre, and M. Yasui, "Determinants of AQP6 trafficking to intracellular sites versus the plasma membrane in transfected mammalian cells," The Biology of the Cell, vol. 98, no. 2, pp. 101-109, 2006.

[27] N. E. Rabaud, L. Song, Y. Wang, P. Agre, M. Yasui, and J. M. Carbrey, "Aquaporin 6 binds calmodulin in a calciumdependent manner," Biochemical and Biophysical Research Communications, vol. 383, no. 1, pp. 54-57, 2009.

[28] L. Lagostena and F. Mammano, "Intracellular calcium dynamics and membrane conductance changes evoked by Deiters' cell purinoceptor activation in the organ of Corti," Cell Calcium, vol. 29, no. 3, pp. 191-198, 2001.

[29] S. Nielsen, C.-L. Chou, D. Marples, E. I. Christensen, B. K. Kishore, and M. A. Knepper, "Vasopressin increases water permeability of kidney collecting duct by inducing translocation of aquaporin-CD water channels to plasma membrane," Proceedings of the National Academy of Sciences of the United States of America, vol. 92, no. 4, pp. 1013-1017, 1995.

[30] R. Nørregaard, B. L. Jensen, C. Li, et al., "COX-2 inhibition prevents downregulation of key renal water and sodium transport proteins in response to bilateral ureteral obstruction," American Journal of Physiology, vol. 289, no. 2, pp. F322-F333, 2005.

[31] U.-R. Heinrich, J. Brieger, O. Selivanova, et al., "COX-2 expression in the guinea pig cochlea is partly altered by moderate sound exposure," Neuroscience Letters, vol. 394, no. 2, pp. 121-126, 2006. 

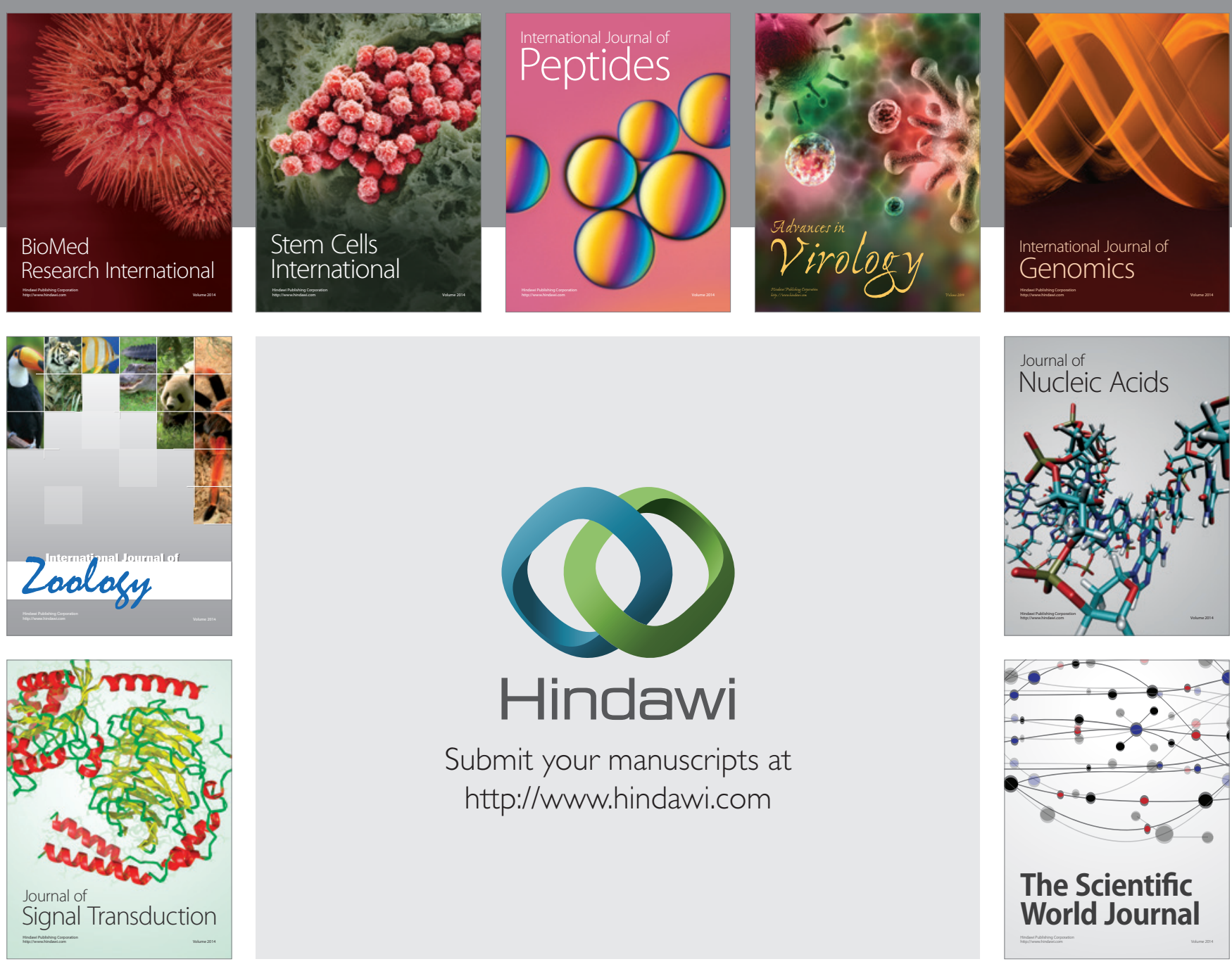

Submit your manuscripts at

http://www.hindawi.com
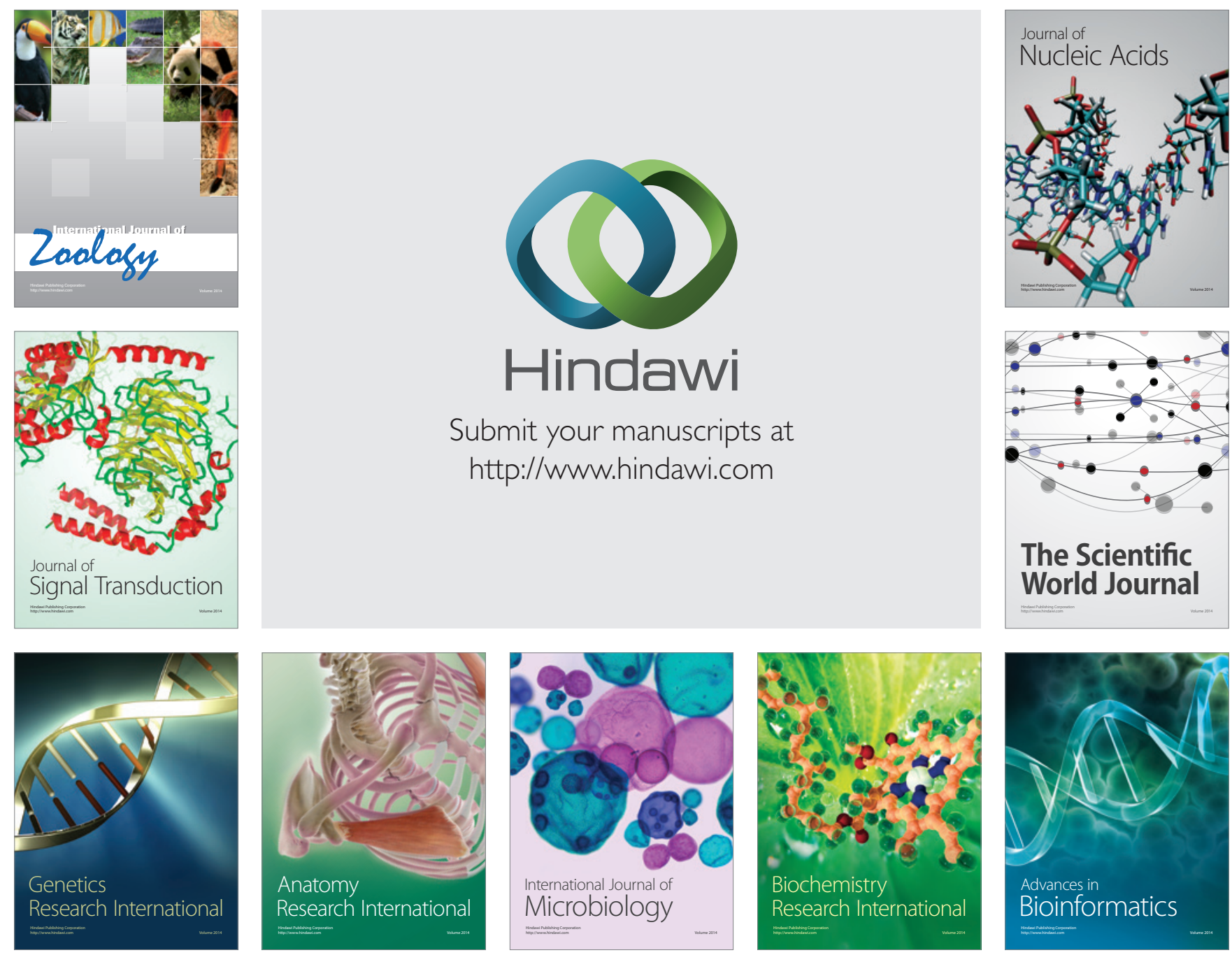

The Scientific World Journal
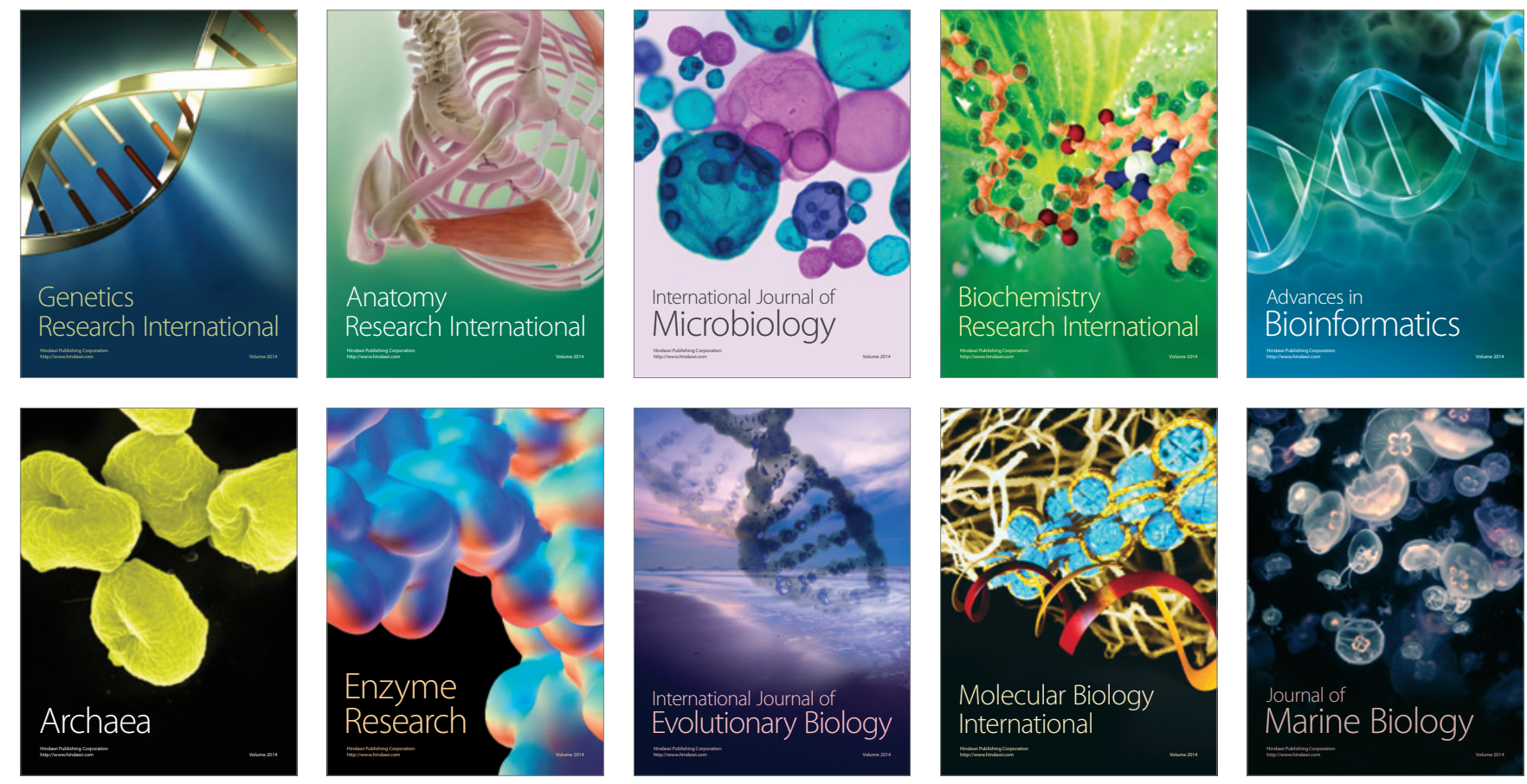